\title{
Effect of Time Delay and Antibodies on HCV Dynamics with Cure Rate and Two Routes of Infection
}

\author{
Ahmed M. Elaiw, Shafeek A. Ghaleb, Aatef Hobiny \\ Department of Mathematics, Faculty of Science, King Abdulaziz University, Jeddah, KSA \\ Email: a_m_elaiw@yahoo.com
}

How to cite this paper: Elaiw, A.M., Ghaleb, S.A. and Hobiny, A. (2018) Effect of Time Delay and Antibodies on HCV Dynamics with Cure Rate and Two Routes of Infection. Journal of Applied Mathematics and Physics, 6, 1120-1138.

https://doi.org/10.4236/jamp.2018.65096

Received: April 22, 2018

Accepted: May 28, 2018

Published: May 31, 2018

Copyright (c) 2018 by authors and Scientific Research Publishing Inc. This work is licensed under the Creative Commons Attribution International License (CC BY 4.0). http://creativecommons.org/licenses/by/4.0/

\begin{abstract}
In this paper we propose and analyze an HCV dynamics model taking into consideration the cure of infected hepatocytes and antibody immune response. We incorporate both virus-to-cell and cell-to-cell transmissions into the model. We incorporate a distributed-time delay to describe the time between the HCV or infected cell contacts an uninfected hepatocyte and the emission of new active HCV. We show that the solutions of the proposed model are nonnegative and ultimately bounded. We derive two threshold parameters which fully determine the existence and stability of the three steady states of the model. Using Lyapunov functionals, we established the global stability of the steady states. The theoretical results are confirmed by numerical simulations.
\end{abstract}

\section{Keywords}

HCV Infection, Distributed Time Delay, Global Stability, Cell-to-Cell Transmission, Lyapunov Function

\section{Introduction}

Hepatitis $\mathrm{C}$ virus is considered one of the dangerous human viruses that infects the liver and causes the lever cirrhosis. Mathematical modeling and analysis of within-host HCV dynamics have been studied by many authors (see e.g. [1]-[12]). These works can help researchers for better understanding the HCV dynamical behavior and providing new suggestions for clinical treatment. Immune response plays an important role in controlling the dynamics of several viruses (see e.g. [13] [14] [15] [16] [17]). Cytotoxic T Lymphocyte (CTL) and antibodies play a central role of immune response. CTL cells attack and kill the 
infected cells. The B cell produces antibodies to neutralize the viruses. Mathematical models of HCV dynamics with antibody immune response have been proposed in [18] [19] [20]. The models presented in [18] [19] [20] assume that an uninfected hepatocyte becomes infected by contacting with HCV (virus-to-cell transmission). It has been reported in [21] [22] [23] that the HCV can also spread by cell-to-cell transmission.

The "cure" of infected cells has been considered in the virus dynamics models in several works (see e.g. [24]-[39]). In [40], both cure and cell-to-cell transmissions have been considered in the virus dynamics model, but without taking the immune response into account. In a very recent paper, Pan and Chakrabarty [41] have proposed the following mathematical model of HCV dynamics which incorporates 1) both virus-to-cell and cell-to-cell transmissions, 2) cure of infected hepatocytes, and 3) antibody immune response:

$$
\begin{gathered}
\dot{s}(t)=\beta-\hat{\delta} s(t)-\alpha_{1} s(t) p(t)-\alpha_{2} s(t) y(t)+\rho y(t), \\
\dot{y}(t)=\alpha_{1} s(t) p(t)+\alpha_{2} s(t) y(t)-\varepsilon y(t)-\rho y(t), \\
\dot{p}(t)=m y(t)-\gamma p(t)-q z(t) p(t), \\
\dot{z}(t)=r z(t) p(t)-\mu z(t),
\end{gathered}
$$

where, $s, y, p$ and $z$ represent the concentration of uninfected hepatocytes, infected hepatocytes, HCV particles and antibodies, respectively. The uninfected hepatocytes are generated at a constant rate $\beta$, die at rate $\hat{\delta} s$, where $\hat{\delta}$ is the natural death rate constant. The infection rate due to both virus-to-cell and cell-to-cell transmissions is given by $\alpha_{1} s p+\alpha_{2} s y$, where $\alpha_{1}$ and $\alpha_{2}$ are constants. The infected hepatocytes die at rate $\varepsilon y$ and cure at rate $\rho$ y, where $\varepsilon$ and $\rho$ are constants. Constant $m$ is the generation rate of the HCV from infected hepatocytes. Antibodies attack the $\mathrm{HCV}$ at rate $q z p$, proliferate at rate $r z p$ and die at rate $\mu z$, where $q, r$ and $\mu$ are constants.

It is assumed in model (1)-(4) that, the hepatocytes can produce HCV particles once they are contacted by HCV or infected cells. However, there is a time period from the moment of the uninfected hepatocytes that are contacted by the $\mathrm{HCV}$ or infected cells and the moment of producing new active HCV particles [10] [11].

The aim of this paper is to study the qualitative behavior of an HCV dynamics model with antibody immune response. We have incorporated distributed time delay and both virus-to-cell and cell-to-cell transmissions. We derive two threshold parameters and establish the global stability of the three steady states of the model using Lyapunov method.

\section{The Model}

We propose the following HCV dynamics model with distributed time delay:

$$
\begin{gathered}
\dot{s}(t)=\beta-\hat{\delta}_{s}(t)-\alpha_{1} s(t) p(t)-\alpha_{2} s(t) y(t)+\rho y(t), \\
\dot{y}(t)=\alpha_{1} s(t) p(t)+\alpha_{2} s(t) y(t)-\varepsilon y(t)-\rho y(t),
\end{gathered}
$$




$$
\begin{gathered}
\dot{p}(t)=m \int_{0}^{h} \rho(\tau) \mathrm{e}^{-\mu_{1} \tau} y(t-\tau) \mathrm{d} \tau-\gamma p(t)-q z(t) p(t), \\
\dot{z}(t)=r z(t) p(t)-\mu z(t) .
\end{gathered}
$$

We assume that, the HCV or infected cell contacts an uninfected hepatocyte at time $t-\tau$, the cell becomes infected at time $t$, where $\tau$ is a distributed parameter over the time interval $[0, h]$. The factors $\mathrm{e}^{-\mu_{1} \tau}$ represents the probability of surviving the hepatocyte during the time delay period, where $\mu_{1}$ is a constant. $\rho(\tau)$ is a probability distribution function satisfying $\rho(\tau)>0$ and

$$
\int_{0}^{h} \rho(\tau) \mathrm{d} \tau=1, \int_{0}^{h} \rho(v) \mathrm{e}^{\varsigma v} \mathrm{~d} v<\infty,
$$

where $\varsigma$ and $h$ are positive constants. Let us denote $\Theta(\tau)=\rho(\tau) \mathrm{e}^{-\mu_{1} \tau}$ and $F=\int \Theta(\tau) \mathrm{d} \tau$, thus $0<F \leq 1$. Let the initial conditions for system (5)-(8) be given as:

$$
\begin{aligned}
& s(\eta)=\zeta_{1}(\eta), y(\eta)=\zeta_{2}(\eta), \\
& p(\eta)=\zeta_{3}(\eta), z(\eta)=\zeta_{4}(\eta), \\
& \zeta_{j}(\eta) \geq 0, \eta \in[-h, 0], \\
& \zeta_{j} \in C\left([-h, 0], \mathbb{R}_{\geq 0}^{4}\right), \quad j=1, \cdots, 4,
\end{aligned}
$$

where $C$ is the Banach space of continuous functions mapping the interval $[-h, 0]$ into $\mathbb{R}_{\geq 0}^{4}$. Then, the uniqueness of the solution for $t>0$ is guaranteed [42].

\subsection{Basic Properties}

In this subsection, we investigate the nonnegativity and boundedness of solutions.

Proposition 1. The solutions of system (5)-(8) with the initial states (9) are nonnegative and ultimately bounded.

Proof. From Equation (5) we have $\left.\dot{s}\right|_{s=0}=\beta+\rho y>0$. Hence, $s(t)>0$ for all $y \geq 0$. Moreover, for all $t \in[0, h]$ we have

$$
\begin{gathered}
y(t)=\zeta_{2}(0) \mathrm{e}^{-(\varepsilon+\rho) t}+\int_{0}^{t} e^{-(\varepsilon+\rho)(t-\eta)}\left[\alpha_{1} s(\eta) p(\eta)+\alpha_{2} s(\eta) y(\eta)\right] \mathrm{d} \eta \geq 0, \\
p(t)=\zeta_{3}(0) \mathrm{e}^{-\int_{0}^{t}(\gamma+q z(\xi)) \mathrm{d} \xi}+m \int_{0}^{t} \mathrm{e}^{-\int_{\eta}^{t}(\gamma+q z(\xi)) \mathrm{d} \xi} \int_{0}^{h} \Theta_{2}(\tau) y(\eta-\tau) \mathrm{d} \tau \mathrm{d} \eta \geq 0, \\
z(t)=\zeta_{4}(0) \mathrm{e}^{-\int_{0}^{t}(\kappa-r p(\xi)) \mathrm{d} \xi} \geq 0 .
\end{gathered}
$$

By recursive argument we get $y(t) \geq 0, p(t) \geq 0$, and $z(t) \geq 0$, for all $t \geq 0$.

Next, we establish the boundedness of the model's solutions. The nonnegativity of the model's solution implies that

$$
\dot{s}(t) \leq \beta-\hat{\delta} s(t)+\rho y(t),
$$


We let $Q_{1}(t)=s(t)+y(t)$, then

$$
\dot{Q}_{1}(t)=\beta-\hat{\delta} s(t)-\varepsilon y(t) \leq \beta-\sigma_{1}(s(t)+y(t))=\beta-\sigma_{1} Q_{1}(t),
$$

where $\sigma_{1}=\min \{\hat{\delta}, \varepsilon\}$. Hence $Q_{1}(t) \leq L_{1}$, if $Q_{1}(t) \leq L_{1}$ where $L_{1}=\frac{\beta}{\sigma_{1}}$. It follows that $s(t) \leq L_{1}$ and $y(t) \leq L_{1} \quad$ if $\quad s(0)+y(0) \leq L_{1}$. Moreover, let $Q_{2}(t)=p(t)+\frac{q}{r} z(t)$, then

$$
\begin{aligned}
\dot{Q}_{2}(t) & =m \int_{0}^{h} \Theta(\tau) y(t-\tau) d \tau-\gamma p(t)-\frac{q \mu}{r} z(t) \leq m L_{1} F-\gamma p(t)-\frac{q \mu}{r} z(t) \\
& \leq m L_{1}-\sigma_{2}\left(p(t)+\frac{q}{r} z(t)\right)=m L_{1}-\sigma_{2} Q_{2}(t),
\end{aligned}
$$

where $\sigma_{2}=\min \{\gamma, \mu\}$. It follows that, $\limsup _{t \rightarrow \infty} Q_{2}(t) \leq L_{2}$, where $L_{2}=\frac{m L_{1}}{\sigma_{2}}$. Since $\quad p(t) \geq 0$ and $\quad z(t) \geq 0$, then $\quad \limsup _{t \rightarrow \infty} p(t) \leq L_{2} \quad$ and $\limsup _{t \rightarrow \infty} z(t) \leq L_{3}$, where $L_{3}=\frac{r}{q} L_{2}$. Therefore, $s(t), y(t), p(t)$ and $z(t)$ are ultimately bounded.

According to Proposition 1, we can show that the region

$$
\Delta=\left\{(s, y, p, z) \in C^{4}:\|s\| \leq L_{1},\|y\| \leq L_{1},\|p\| \leq L_{2},\|z\| \leq L_{3}\right\},
$$

is positively invariant with respect to system (5)-(8).

\subsection{The Steady States and Threshold Parameters}

Lemma 1. For system (5)-(8) there exist two threshold parameters $R_{0}>0$, and $R_{1}^{z}>0$, such that

1) if $R_{0} \leq 1$, then there exists only one steady state $\Pi_{0}$,

2) if $R_{1}^{z} \leq 1<R_{0}$, then there exist only two steady states $\Pi_{0}$ and $\Pi_{1}$,

3) if $R_{0}>1$ and $R_{1}^{z}>1$, then there exist three steady states $\Pi_{0}, \Pi_{1}$ and $\Pi_{2}$.

Proof. Let $(s, y, p, z)$ be any steady state satisfying

$$
\begin{gathered}
\beta-\hat{\delta}_{s}-\alpha_{1} s p-\alpha_{2} s y+\rho y=0, \\
\alpha_{1} s p+\alpha_{2} s y-\varepsilon y-\rho y=0, \\
m F y-\gamma p-q z p=0, \\
(r p-\mu) z=0 .
\end{gathered}
$$

We find that system (10)-(13) admits three steady states.

1) Infection-free steady state $\Pi_{0}=\left(s_{0}, 0,0,0\right)$, where $s_{0}=\beta / \hat{\delta}$.

2) Chronic-infection steady state without immune response $\Pi_{1}=\left(s_{1}, y_{1}, p_{1}, 0\right)$, where

$$
s_{1}=\frac{(\varepsilon+\rho) \gamma}{F m \alpha_{1}+\gamma \alpha_{2}}
$$




$$
\begin{gathered}
y_{1}=\frac{\hat{\delta} s_{1}}{\varepsilon}\left(\frac{\beta\left(F m \alpha_{1}+\gamma \alpha_{2}\right)}{\hat{\delta} \gamma(\varepsilon+\rho)}-1\right), \\
p_{1}=\frac{F m y_{1}}{\gamma} .
\end{gathered}
$$

Clearly $\Pi_{1}$ exists if

$$
\frac{\beta\left(F m \alpha_{1}+\gamma \alpha_{2}\right)}{\hat{\delta} \gamma(\varepsilon+\rho)}>1
$$

Let us define

$$
R_{0}=\frac{\beta\left(F m \alpha_{1}+\gamma \alpha_{2}\right)}{\hat{\delta} \gamma(\varepsilon+\rho)},
$$

In terms of $R_{0}$, we can write the steady state components for $\Pi_{1}$ as:

$$
\begin{gathered}
s_{1}=\frac{s_{0}}{R_{0}}, \quad y_{1}=\frac{\hat{\delta} s_{1}}{\varepsilon}\left(R_{0}-1\right), \\
p_{1}=\frac{F m \hat{\delta} s_{1}}{\gamma \varepsilon}\left(R_{0}-1\right) .
\end{gathered}
$$

3) Chronic-infection steady state with humoral immune $\Pi_{2}=\left(s_{2}, y_{2}, p_{2}, z_{2}\right)$, where

$$
\begin{aligned}
& s_{2}=\frac{r y_{2}(\varepsilon+\rho)}{\mu \alpha_{1}+r y_{2} \alpha_{2}}, \quad y_{2}=\frac{-B+\sqrt{B^{2}-4 A C}}{2 A}, \\
& p_{2}=\frac{\mu}{r}, \quad z_{2}=\frac{\gamma}{q}\left(\frac{r m F y_{2}}{\mu \gamma}-1\right) .
\end{aligned}
$$

where

$$
\begin{aligned}
& A=r \alpha_{2} \varepsilon, \\
& B=\mu \varepsilon \alpha_{1}-r \beta \alpha_{2}+r \hat{\delta}(\varepsilon+\rho), \\
& C=-\beta \mu \alpha_{1} .
\end{aligned}
$$

We note that $\Pi_{2}$ exists when $\frac{r F_{2} m y_{2}}{\mu \gamma}>1$. Now we define

$$
R_{1}^{z}=\frac{r F m y_{2}}{\mu \gamma}=\frac{F m y_{2}}{p_{2} \gamma}
$$

Then $z_{2}=\frac{\gamma}{q}\left(R_{1}^{z}-1\right)$. We define the basic reproduction number for the humoral immune response $R_{\text {Hum }}$ which comes from the limiting (linearized) $z$-dynamics near $z=0$ as:

$$
R_{H u m}^{z}=\frac{p_{1}}{p_{2}}
$$

Lemma 2 1) if $R_{1}^{z}<1$, then $R_{\text {Hum }}^{z}<1$,

2) if $R_{1}^{z}>1$, then $R_{H u m}^{z}>1$, 
3) if $R_{1}^{z}=1$ then $R_{\text {Hum }}^{z}=1$.

Proof. 1) Let $R_{1}^{z}<1$, then from Equation (16) we have $y_{2}<\frac{\gamma p_{2}}{m F}$, and then using Equation (14) we get

$$
\frac{-B+\sqrt{B^{2}-4 A C}}{2 A}<\frac{\gamma p_{2}}{F m},
$$

that leads to

$$
\left(\frac{2 A \gamma p_{2}}{F m}+B\right)^{2}-\left(B^{2}-4 A C\right)>0
$$

Using Equation (15), we can get

$$
\frac{4 \alpha_{2} \varepsilon^{2} \mu^{2} \gamma\left(F m \alpha_{1}+\gamma \alpha_{2}\right)}{m^{2} F^{2}}\left(1-R_{H u m}^{z}\right)>0
$$

then

$$
R_{H u m}^{z}=\frac{r F m s_{1}\left(R_{0}-1\right) \hat{\delta}}{\mu \varepsilon \gamma}<1 .
$$

then $R_{H u m}^{z}<1$. Similarly, one can proof 2) and 3) $\square$.

\section{Global Stability}

The following theorems investigate the global stability of the steady states of system (5)-(8). Let us define the function $H:(0, \infty) \rightarrow[0, \infty)$ as $H(\ell)=\ell-1-\ln \ell$. Denote $(s, y, p, z)=(s(t), y(t), p(t), z(t))$.

Theorem 1. Suppose that $R_{0} \leq 1$, then the infection-free steady state $\Pi_{0}$ is globally asymptotically stable (GAS).

Proof. Constructing a Lyapunov functional

$$
\begin{aligned}
L_{0}(s, y, p, z)= & s_{0} H\left(\frac{s}{s_{0}}\right)+y+\frac{\alpha_{1} s_{0}}{\gamma} p+\frac{q \alpha_{1} s_{0}}{r \gamma} z \\
& +\frac{\rho}{2(\hat{\delta}+\varepsilon) s_{0}}\left[\left(s-s_{0}\right)+y\right]^{2} \\
& +\frac{m \alpha_{1} s_{0}}{\gamma} \int_{0}^{h} \Theta(\tau) \int_{t-\tau}^{t} y(\eta) \mathrm{d} \eta \mathrm{d} \tau .
\end{aligned}
$$

We calculate $\frac{\mathrm{d} L_{0}}{\mathrm{~d} t}$ along the solutions of model (5)-(8) as:

$$
\begin{aligned}
\frac{\mathrm{d} L_{0}}{\mathrm{~d} t}= & \left(1-\frac{s_{0}}{s}\right)\left(\beta-\hat{\delta} s-\alpha_{1} s p-\alpha_{2} s y+\rho y\right)+\alpha_{1} s p+\alpha_{2} s y \\
& -\varepsilon y-\rho y+\frac{\alpha_{1} s_{0}}{\gamma}\left(m \int_{0}^{h} \Theta(\tau) y(t-\tau) \mathrm{d} \tau-\gamma p-q z p\right) \\
& +\frac{q \alpha_{1} s_{0}}{r \gamma}(r z p-\mu z)+\frac{\rho}{(\hat{\delta}+\varepsilon) s_{0}}\left[\left(s-s_{0}\right)+y\right](\beta-\hat{\delta} s-\varepsilon y) \\
& +\frac{m \alpha_{1} s_{0}}{\gamma} \int_{0}^{h} \Theta(\tau)[y-y(t-\tau)] \mathrm{d} \tau .
\end{aligned}
$$


Collecting terms of Equation (17) and using $\beta=\hat{\delta} s_{0}$ we obtain

$$
\begin{aligned}
\frac{\mathrm{d} L_{0}}{\mathrm{~d} t}= & \left(1-\frac{s_{0}}{s}\right)\left(\hat{\delta} s_{0}-\hat{\delta} s\right)+\alpha_{2} s_{0} y+\left(1-\frac{s_{0}}{s}\right) \rho y \\
& -(\varepsilon+\rho) y+\frac{\alpha_{1} s_{0} F}{\gamma} m y-\frac{q \alpha_{1} s_{0}}{r \gamma} \mu z \\
& -\frac{\rho}{(\hat{\delta}+\varepsilon) s_{0}}\left[\left(s-s_{0}\right)+y\right]\left(\hat{\delta}\left(s-s_{0}\right)+\varepsilon y\right) .
\end{aligned}
$$

We note that

$$
\left(1-\frac{s_{0}}{s}\right) \rho y=-\frac{\rho y}{s s_{0}}\left(s-s_{0}\right)^{2}+\frac{\rho y}{s_{0}}\left(s-s_{0}\right) .
$$

Therefore

$$
\begin{aligned}
\frac{\mathrm{d} L_{0}}{\mathrm{~d} t}= & -\hat{\delta} \frac{\left(s-s_{0}\right)^{2}}{s}+\alpha_{2} s_{0} y-\frac{\rho y}{s s_{0}}\left(s-s_{0}\right)^{2}+\frac{\rho y}{s_{0}}\left(s-s_{0}\right) \\
& -(\varepsilon+\rho) y+\frac{\alpha_{1} s_{0} F}{\gamma} m y-\frac{q \alpha_{1} s_{0}}{r \gamma} \mu z-\frac{\rho \hat{\delta}\left(s-s_{0}\right)^{2}}{(\hat{\delta}+\varepsilon) s_{0}} \\
& -\frac{\rho \varepsilon\left(s-s_{0}\right) y}{(\hat{\delta}+\varepsilon) s_{0}}-\frac{\rho \hat{\delta}\left(s-s_{0}\right) y}{(\hat{\delta}+\varepsilon) s_{0}}-\frac{\rho \varepsilon y^{2}}{(\hat{\delta}+\varepsilon) s_{0}} \\
= & -\left(\hat{\delta} s_{0}+\rho y+\frac{\rho \hat{\delta} s}{\hat{\delta}+\varepsilon)}\right) \frac{\left(s-s_{0}\right)^{2}}{s s_{0}}-\frac{\rho \varepsilon y^{2}}{(\hat{\delta}+\varepsilon) s_{0}} \\
& -\frac{q \alpha_{1} s_{0}}{r \gamma} \mu z+(\varepsilon+\rho)\left(\frac{\left(m \alpha_{1} F+\gamma \alpha_{2}\right) s_{0}}{\gamma(\varepsilon+\rho)}-1\right) y \\
= & -\left(\hat{\delta} s_{0}+\rho y+\frac{\rho \hat{\delta} s}{\hat{\delta}+\varepsilon}\right) \frac{\left(s-s_{0}\right)^{2}}{s s_{0}}-\frac{\rho \varepsilon y^{2}}{(\hat{\delta}+\varepsilon) s_{0}} \\
& -\frac{q \alpha_{1} s_{0}}{r \gamma} \mu z+(\varepsilon+\rho)\left(R_{0}-1\right) y .
\end{aligned}
$$

Since $R_{0} \leq 1$, then $\frac{\mathrm{d} L_{0}}{\mathrm{~d} t} \leq 0$ for all $s, y, p, z>0$. Moreover $\frac{\mathrm{d} L_{0}}{\mathrm{~d} t}=0$ if and only if $s(t)=s(0), y(t)=z(t)=0$. Let $\Gamma_{0}=\left\{(s, y, p, z): \frac{\mathrm{d} L_{0}}{\mathrm{~d} t}=0\right\}$ and $\Gamma_{0}$ be the largest invariant subset of $\Gamma_{0}$. The solution of system (5)-(8) tend to $\Gamma_{0}$. For each element of $\Gamma_{0}$ we have $y(t)=0$, then $\dot{y}(t)$ and Equation (6) we get

$$
y(t)=0=\alpha_{1} s_{0} p(t)
$$

Then $p(t)=0$. It follows that $\Gamma_{0}$ contains a single point that is $\left\{\Gamma_{0}\right\}$. Appling LaSalle's invariance principle (LIP), we get that $\Pi_{0}$ is GAS.

Theorem 2. Suppose that $R_{1}^{z} \leq 1<R_{0} \leq 1+\frac{\rho}{\varepsilon}$, then $\Pi_{1}$ is GAS.

Proof. Let us define a function $L_{1}(s, y, p, z)$ as: 


$$
\begin{aligned}
L_{1}= & s_{1} H\left(\frac{s}{s_{1}}\right)+y_{1} H\left(\frac{y}{y_{1}}\right)+\frac{\alpha_{1} s_{1} p_{1}}{F m y_{1}} p_{1} H\left(\frac{p}{p_{1}}\right) \\
& +\frac{\alpha_{1} s_{1} p_{1} q}{F m y_{1} r} z+\frac{\rho}{2(\hat{\delta}+\varepsilon) s_{1}}\left[\left(s-s_{1}\right)+\left(y-y_{1}\right)\right]^{2} \\
& +\frac{\alpha_{1} s_{1} p_{1}}{F} \int_{0}^{h} \Theta(\tau) \int_{t-\tau}^{t} H\left(\frac{y(\eta)}{y_{1}}\right) \mathrm{d} \eta \mathrm{d} \tau .
\end{aligned}
$$

Calculating $\frac{\mathrm{d} L_{1}}{\mathrm{~d} t}$ along the trajectories of system (5)-(8), we get

$$
\begin{aligned}
\frac{\mathrm{d} L_{1}}{\mathrm{~d} t}= & \left(1-\frac{s_{1}}{s}\right)\left(\beta-\hat{\delta} s-\alpha_{1} s p-\alpha_{2} s y+\rho y\right) \\
& +\left(1-\frac{y_{1}}{y}\right)\left(\alpha_{1} s p+\alpha_{2} s y-\varepsilon y-\rho y\right) \\
& +\frac{\alpha_{1} s_{1} p_{1}}{F m y_{1}}\left(1-\frac{p_{1}}{p}\right)\left(m \int_{0}^{h} \Theta(\tau) y(t-\tau) \mathrm{d} \tau-\gamma p-q z p\right) \\
& +\frac{\alpha_{1} s_{1} p_{1} q}{F m y_{1} r}(r z p-\mu z)+\frac{\rho}{(\hat{\delta}+\varepsilon) s_{1}}\left[\left(s-s_{1}\right)+\left(y-y_{1}\right)\right](\beta-\hat{\delta} s-\varepsilon y) . \\
& +\frac{\alpha_{1} s_{1} p_{1}}{F} \int_{0}^{h} \Theta(\tau)\left[\frac{y}{y_{1}}-\frac{y(t-\tau)}{y_{1}}+\ln \left(\frac{y(t-\tau)}{y}\right)\right] \mathrm{d} \tau .
\end{aligned}
$$

Collecting terms of Equation (19), we get

$$
\begin{aligned}
\frac{\mathrm{d} L_{1}}{\mathrm{~d} t}= & \left(1-\frac{s_{1}}{s}\right)(\beta-\hat{\delta} s+\rho y)+\alpha_{1} s_{1} p+\alpha_{2} s_{1} y-(\varepsilon+\rho) y_{1} \frac{y}{y_{1}} \\
& -\left(\alpha_{1} s p+\alpha_{2} s y\right) \frac{y_{1}}{y}+(\varepsilon+\rho) y_{1}-\frac{\alpha_{1} s_{1} p_{1}}{F m y_{1}} \gamma p_{1} \frac{p}{p_{1}} \\
& -\frac{\alpha_{1} s_{1} p_{1}}{F y_{1}} \int_{0}^{h} \Theta(\tau) y(t-\tau) \frac{p_{1}}{p} \mathrm{~d} \tau+\frac{\alpha_{1} s_{1} p_{1}}{F m y_{1}} \gamma p_{1}+\frac{\alpha_{1} s_{1} p_{1}}{F m y_{1}} q z p_{1} \\
& -\frac{\alpha_{1} s_{1} p_{1} q}{F m y_{1} r} \mu z+\frac{\rho}{(\hat{\delta}+\varepsilon) s_{1}}\left[\left(s-s_{1}\right)+\left(y-y_{1}\right)\right](\beta-\hat{\delta} s-\varepsilon y) \\
& +\frac{\alpha_{1} s_{1} p_{1}}{y_{1}} y+\frac{\alpha_{1} s_{1} p_{1}}{F} \int_{0}^{h} \Theta(\tau) \ln \left(\frac{y(t-\tau)}{y}\right) \mathrm{d} \tau .
\end{aligned}
$$

Applying condition of equilibrum $\Pi_{1}$ :

$$
\begin{gathered}
\beta=\hat{\delta}_{1}+\alpha_{1} s_{1} p_{1}+\alpha_{2} s_{1} y_{1}-\rho y_{1}=\hat{\delta} s_{1}+\varepsilon y_{1}, \\
p_{1}=\frac{F m}{\gamma} y_{1},(\varepsilon+\rho) y_{1}=\alpha_{1} s_{1} p_{1}+\alpha_{2} s_{1} y_{1}
\end{gathered}
$$

we get

$$
\begin{aligned}
\frac{\mathrm{d} L_{1}}{\mathrm{~d} t}= & -\hat{\delta} \frac{\left(s-s_{1}\right)^{2}}{s}+\left(\alpha_{1} s_{1} p_{1}+\alpha_{2} s_{1} y_{1}\right)\left(1-\frac{s_{1}}{s}\right)+\rho\left(y-y_{1}\right)\left(1-\frac{s_{1}}{s}\right) \\
& +\alpha_{1} s_{1} p_{1} \frac{p}{p_{1}}+\alpha_{2} s_{1} y_{1} \frac{y}{y_{1}}-\left(\alpha_{1} s_{1} p_{1}+\alpha_{2} s_{1} y_{1}\right) \frac{y}{y_{1}}-\alpha_{1} s_{1} p_{1} \frac{s p}{s_{1} p_{1}} \frac{y_{1}}{y} \\
& -\alpha_{2} s_{1} y_{1} \frac{s}{s_{1}}+\left(\alpha_{1} s_{1} p_{1}+\alpha_{2} s_{1} y_{1}\right)-\alpha_{1} s_{1} p_{1} \frac{p}{p_{1}}
\end{aligned}
$$




$$
\begin{aligned}
& -\frac{\alpha_{1} s_{1} p_{1}}{F} \int_{0}^{h} \Theta(\tau) \frac{y(t-\tau)}{y_{1}} \frac{p_{1}}{p} \mathrm{~d} \tau+\alpha_{1} s_{1} p_{1}+\frac{\alpha_{1} s_{1}}{\gamma} q z p_{1}-\frac{q \alpha_{1} s_{1} \mu}{r \gamma} z \\
& +\frac{\rho}{(\hat{\delta}+\varepsilon) s_{1}}\left[\left(s-s_{1}\right)+\left(y-y_{1}\right)\right]\left(\hat{\delta}\left(s_{1}-s\right)+\alpha_{1} s_{1} p_{1}+\alpha_{2} s_{1} y_{1}-\rho y_{1}-\varepsilon y\right) \\
& +\alpha_{1} s_{1} p_{1} \frac{y}{y_{1}}+\frac{\alpha_{1} s_{1} p_{1}}{F} \int_{0}^{h} \Theta(\tau) \ln \left(\frac{y(t-\tau)}{y}\right) \mathrm{d} \tau .
\end{aligned}
$$

thus

$$
\begin{aligned}
\frac{\mathrm{d} L_{1}}{\mathrm{~d} t}= & -\hat{\delta} \frac{\left(s-s_{1}\right)^{2}}{s}+\alpha_{1} s_{1} p_{1}\left(1-\frac{s_{1}}{s}\right)+\rho\left(y-y_{1}\right)\left(1-\frac{s_{1}}{s}\right) \\
& +\alpha_{2} s_{1} y_{1}\left(2-\frac{s_{1}}{s}-\frac{s}{s_{1}}\right)-\alpha_{1} s_{1} p_{1} \frac{s p}{s_{1} p_{1}} \frac{y_{1}}{y}+2 \alpha_{1} s_{1} p_{1} \\
& -\frac{\alpha_{1} s_{1} p_{1}}{F y_{1}} \int_{0}^{h} \Theta(\tau) y(t-\tau) \frac{p_{1}}{p} \mathrm{~d} \tau+\frac{\alpha_{1} s_{1}}{\gamma} q z p_{1}-\frac{q \alpha_{1} s_{1} \mu}{r \gamma} z \\
& -\frac{\rho}{(\hat{\delta}+\varepsilon) s_{1}}\left[\left(s-s_{1}\right)+\left(y-y_{1}\right)\right]\left(\hat{\delta}\left(s-s_{1}\right)+\varepsilon\left(y-y_{1}\right)\right) \\
& +\frac{\alpha_{1} s_{1} p_{1}}{F} \int_{0}^{h} \Theta(\tau) \ln \left(\frac{y(t-\tau)}{y}\right) \mathrm{d} \tau .
\end{aligned}
$$

We note that

$$
\rho\left(y-y_{1}\right)\left(1-\frac{s_{1}}{s}\right)=-\frac{\rho\left(y-y_{1}\right)}{s s_{1}}\left(s-s_{1}\right)^{2}+\frac{\rho\left(y-y_{1}\right)}{s_{1}}\left(s-s_{1}\right) .
$$

Then

$$
\begin{aligned}
& \frac{\mathrm{d} L_{1}}{\mathrm{~d} t}=-\hat{\delta} \frac{\left(s-s_{1}\right)^{2}}{s}+\alpha_{1} s_{1} p_{1}\left(1-\frac{s_{1}}{s}\right)-\frac{\rho\left(y-y_{1}\right)}{s s_{1}}\left(s-s_{1}\right)^{2} \\
& +\frac{\rho\left(y-y_{1}\right)}{s_{1}}\left(s-s_{1}\right)+\alpha_{2} s_{1} y_{1}\left(2-\frac{s_{1}}{s}-\frac{s}{s_{1}}\right)-\alpha_{1} s_{1} p_{1} \frac{s p}{s_{1} p_{1}} \frac{y_{1}}{y} \\
& +2 \alpha_{1} s_{1} p_{1}-\frac{\alpha_{1} s_{1} p_{1}}{F} \int_{0}^{h} \Theta(\tau) \frac{y(t-\tau)}{y_{1}} \frac{p_{1}}{p} \mathrm{~d} \tau+\frac{q \alpha_{1} s_{1}}{\gamma}\left(p_{1}-p_{2}\right) z \\
& -\frac{\rho}{(\hat{\delta}+\varepsilon) s_{1}}\left[\left(s-s_{1}\right)+\left(y-y_{1}\right)\right]\left(\hat{\delta}\left(s-s_{1}\right)+\varepsilon\left(y-y_{1}\right)\right) \\
& +\frac{\alpha_{1} s_{1} p_{1}}{F} \int_{0}^{h} \Theta(\tau) \ln \left(\frac{y(t-\tau)}{y}\right) \mathrm{d} \tau \\
& =-\left(\hat{\delta}_{1}+\rho\left(y-y_{1}\right)+\frac{\rho \hat{\delta} s}{\hat{\delta}+\varepsilon}\right) \frac{\left(s-s_{1}\right)^{2}}{s s_{1}}-\frac{\rho \varepsilon\left(y-y_{1}\right)^{2}}{(\hat{\delta}+\varepsilon) s_{1}}+\alpha_{1} s_{1} p_{1}\left(1-\frac{s_{1}}{s}\right) \\
& +\frac{\rho\left(y-y_{1}\right)}{s_{1}}\left(s-s_{1}\right)+\alpha_{2} s_{1} y_{1}\left(2-\frac{s_{1}}{s}-\frac{s}{s_{1}}\right)-\alpha_{1} s_{1} p_{1} \frac{s p}{s_{1} p_{1}} \frac{y_{1}}{y}+2 \alpha_{1} s_{1} p_{1} \\
& -\frac{\alpha_{1} s_{1} p_{1}}{F} \int_{0}^{h} \Theta(\tau) \frac{y(t-\tau)}{y_{1}} \frac{p_{1}}{p} \mathrm{~d} \tau+\frac{q \alpha_{1} s_{1}}{\gamma}\left(p_{1}-p_{2}\right) z-\frac{\rho \hat{\delta}\left(s-s_{1}\right)\left(y-y_{1}\right)}{(\hat{\delta}+\varepsilon) s_{1}} \\
& -\frac{\rho \varepsilon\left(s-s_{1}\right)\left(y-y_{1}\right)}{(\hat{\delta}+\varepsilon) s_{1}}+\frac{\alpha_{1} s_{1} p_{1}}{F} \int_{0}^{h} \Theta(\tau) \ln \left(\frac{y(t-\tau)}{y}\right) \mathrm{d} \tau .
\end{aligned}
$$


Consider the following equalities

$$
\ln \left(\frac{y(t-\tau)}{y}\right)=\ln \left(\frac{p_{i} y(t-\tau)}{p y_{i}}\right)+\ln \left(\frac{s p y_{i}}{s_{i} p_{i} y}\right)+\ln \left(\frac{s_{i}}{s}\right), \quad i=1,2 .
$$

Simplify Equation (20) and let $i=1$, in Equation(21) we get

$$
\begin{aligned}
\frac{\mathrm{d} L_{1}}{\mathrm{~d} t}= & -\left(\hat{\delta} s_{1}+\rho\left(y-y_{1}\right)+\frac{\rho \hat{\delta} s}{\hat{\delta}+\varepsilon}\right) \frac{\left(s-s_{1}\right)^{2}}{s s_{1}}-\frac{\rho \varepsilon\left(y-y_{1}\right)^{2}}{(\hat{\delta}+\varepsilon) s_{1}} \\
& -\alpha_{1} s_{1} p_{1}\left(\frac{s_{1}}{s}-1-\ln \left(\frac{s_{1}}{s}\right)\right)+\alpha_{2} s_{1} y_{1}\left(2-\frac{s_{1}}{s}-\frac{s}{s_{1}}\right) \\
& -\alpha_{1} s_{1} p_{1}\left[\frac{s p y_{1}}{s_{1} p_{1} y}-1-\ln \left(\frac{s p y_{1}}{s_{1} p_{1} y}\right)\right] \\
& -\frac{\alpha_{1} s_{1} p_{1}}{F} \int_{0}^{h} \Theta(\tau)\left[\frac{p_{1} y(t-\tau)}{p y_{1}}-1-\ln \left(\frac{p_{1} y(t-\tau)}{p y_{1}}\right)\right] \mathrm{d} \tau \\
& +\frac{q \alpha_{1} s_{1}}{\gamma}\left(p_{1}-p_{2}\right) z .
\end{aligned}
$$

Equation (22) can be rewrite as:

$$
\begin{aligned}
\frac{\mathrm{d} L_{1}}{\mathrm{~d} t}= & -\left(\hat{\delta} s_{1}+\rho\left(y-y_{1}\right)+\frac{\rho \hat{\delta} s}{\hat{\delta}+\varepsilon}\right) \frac{\left(s-s_{1}\right)^{2}}{s s_{1}}-\frac{\rho \varepsilon\left(y-y_{1}\right)^{2}}{(\hat{\delta}+\varepsilon) s_{1}} \\
& -\alpha_{1} s_{1} p_{1} H\left(\frac{s}{s_{1}}\right)+\alpha_{2} s_{1} y_{1}\left(2-\frac{s_{1}}{s}-\frac{s}{s_{1}}\right)-\alpha_{1} s_{1} p_{1} H\left(\frac{s p y_{1}}{s_{1} p_{1} y}\right) \\
& -\frac{\alpha_{1} s_{1} p_{1}}{F} \int_{0}^{h} \Theta(\tau) H\left(\frac{p_{1} y(t-\tau)}{p y_{1}}\right) \mathrm{d} \tau+\frac{q \alpha_{1} s_{1}}{\gamma}\left(p_{1}-p_{2}\right) z .
\end{aligned}
$$

We note that

$$
\hat{\delta} s_{1}-\rho y_{1}=\frac{\hat{\delta} \rho s_{0}}{\varepsilon R_{0}}\left[\left(1+\frac{\rho}{\varepsilon}\right)-R_{0}\right]
$$

From Lemma 2 we have $p_{1} \leq p_{2}$, then, $\frac{\mathrm{d} L_{1}}{\mathrm{~d} t} \leq 0$ for all $s_{1}, y_{1}$ and $p_{1}>0$, where $\frac{\mathrm{d} L_{1}}{\mathrm{~d} t}=0$ if and only if $s=s_{1}, y=y_{1}, p=p_{1}$ and $z=0$. Thus, the global asymptotic stability of $\Pi_{1}$ follows from LIP when $R_{1}^{z} \leq 1$, and $1<R_{0} \leq 1+\frac{\rho}{\varepsilon}$.

Theorem 3. Suppose that $R_{1}^{z}>1$ and $\hat{\delta}_{s_{2}}-\rho y_{2} \geq 0$, then $\Pi_{2}$ is GAS.

Proof. Define a function $L_{2}(s, y, p, z)$ as:

$$
\begin{aligned}
L_{2}= & s_{2} H\left(\frac{s}{s_{2}}\right)+y_{2} H\left(\frac{y}{y_{2}}\right)+\frac{\alpha_{1} s_{2} p_{2}}{F m y_{2}} p_{2} H\left(\frac{p}{p_{2}}\right)+\frac{q \alpha_{1} s_{2} p_{2}}{F r m y_{2}} z_{2} H\left(\frac{z}{z_{2}}\right) \\
& +\frac{\rho}{2(\hat{\delta}+\varepsilon) s_{2}}\left[\left(s-s_{2}\right)+\left(y-y_{2}\right)\right]^{2}+\frac{\alpha_{1} s_{2} p_{2}}{F} \int_{0}^{h} \Theta(\tau) \int_{t-\tau}^{t} H\left(\frac{y(\eta)}{y_{2}}\right) \mathrm{d} \eta \mathrm{d} \tau .
\end{aligned}
$$


Calculating $\frac{\mathrm{d} L_{2}}{\mathrm{~d} t}$ as:

$$
\begin{aligned}
\frac{\mathrm{d} L_{2}}{\mathrm{~d} t}= & \left(1-\frac{s_{2}}{s}\right)\left(\beta-\hat{\delta} s-\alpha_{1} s p-\alpha_{2} s y+\rho y\right) \\
& +\left(1-\frac{y_{2}}{y}\right)\left(\alpha_{1} s p+\alpha_{2} s y-\varepsilon y-\rho y\right) \\
& +\frac{\alpha_{1} s_{2} p_{2}}{F m y_{2}}\left(1-\frac{p_{2}}{p}\right)\left(m \int_{0}^{h} \Theta(\tau) y(t-\tau) \mathrm{d} \tau-\gamma p-q z p\right) \\
& +\frac{q \alpha_{1} s_{2} p_{2}}{F r m y_{2}}\left(1-\frac{z_{2}}{z}\right)(r z p-\mu z) \\
& +\frac{\rho}{(\hat{\delta}+\varepsilon) s_{2}}\left[\left(s-s_{2}\right)+\left(y-y_{2}\right)\right](\beta-\hat{\delta} s-\varepsilon y) \\
& +\frac{\alpha_{1} s_{2} p_{2}}{F} \int_{0}^{h} \Theta(\tau)\left[\frac{y}{y_{2}}-\frac{y(t-\tau)}{y_{2}}+\ln \left(\frac{y(t-\tau)}{y}\right)\right] \mathrm{d} \tau .
\end{aligned}
$$

Collecting terms of Equation (24) and applying the equilibrium conditions for $\Pi_{2}$ :

$$
\begin{gathered}
\beta=\hat{\delta} s_{2}+\alpha_{1} s_{2} p_{2}+\alpha_{2} s_{2} y_{2}-\rho y_{2}=\hat{\delta} s_{2}+\varepsilon y_{2}, \\
p_{2}=\frac{\mu}{r},(\varepsilon+\rho) y_{2}=\alpha_{1} s_{2} p_{2}+\alpha_{2} s_{2} y_{2} \\
\varepsilon y_{2}=\frac{\varepsilon}{m F}\left(\gamma p_{2}+q p_{2} z_{2}\right) .
\end{gathered}
$$

we get

$$
\begin{aligned}
\frac{\mathrm{d} L_{2}}{\mathrm{~d} t}= & -\hat{\delta} \frac{\left(s-s_{2}\right)^{2}}{s}+\left(\alpha_{1} s_{2} p_{2}+\alpha_{2} s_{2} y_{2}+\rho\left(y-y_{2}\right)\right)\left(1-\frac{s_{2}}{s}\right)+\alpha_{1} s_{2} p+\alpha_{2} s_{2} y \\
& -\left(\alpha_{1} s_{2} p_{2}+\alpha_{2} s_{2} y_{2}\right) \frac{y}{y_{2}}-\alpha_{1} s_{2} p_{2} \frac{s p}{s_{2} p_{2}} \frac{y_{2}}{y}-\alpha_{2} s_{2} y_{2} \frac{s}{s_{2}}+\left(\alpha_{1} s_{2} p_{2}+\alpha_{2} s_{2} y_{2}\right) \\
& -\frac{\alpha_{1} s_{2} p_{2}}{F m y_{2}}\left(F m y_{2}-q p_{2} z_{2}\right) \frac{p}{p_{2}}-\frac{\alpha_{1} s_{2} p_{2}}{F} \int_{0}^{h} \Theta(\tau) \frac{y(t-\tau)}{y_{2}} \frac{p_{2}}{p} \mathrm{~d} \tau \\
& +\frac{\alpha_{1} s_{2} p_{2}}{F m y_{2}}\left(F m y_{2}-q p_{2} z_{2}\right)-\frac{q \alpha_{1} s_{2} p_{2}}{F m y_{2}} z_{2} p+\frac{q \alpha_{1} s_{2} p_{2}}{F r m y_{2}} \mu z_{2} \\
& +\frac{\rho}{(\hat{\delta}+\varepsilon) s_{2}}\left[\left(s-s_{2}\right)+\left(y-y_{2}\right)\right]\left(\hat{\delta}\left(s_{2}-s\right)+\alpha_{1} s_{2} p_{2}+\alpha_{2} s_{2} y_{2}-\rho y_{2}-\varepsilon y\right) \\
& +\frac{\alpha_{1} s_{2} p_{2}}{y_{2}} y+\frac{\alpha_{1} s_{2} p_{2}}{F} \int_{0}^{h} \Theta(\tau) \ln \left(\frac{y(t-\tau)}{y}\right) \mathrm{d} \tau \\
= & -\hat{\delta} \frac{\left(s-s_{2}\right)^{2}}{s}+\left(\alpha_{1} s_{2} p_{2}+\alpha_{2} s_{2} y_{2}\right)\left(1-\frac{s_{2}}{s}\right)+\rho\left(y-y_{2}\right)\left(1-\frac{s_{2}}{s}\right)+\alpha_{1} s_{2} p_{2} \frac{p}{p_{2}} \\
+ & \alpha_{2} s_{2} y_{2} \frac{y}{y_{2}}-\left(\alpha_{1} s_{2} p_{2}+\alpha_{2} s_{2} y_{2}\right) \frac{y}{y_{2}}-\alpha_{1} s_{2} p_{2} \frac{s p}{s_{2} p_{2}} \frac{y_{2}}{y}-\alpha_{2} s_{2} y_{2} \frac{s}{s_{2}} \\
+ & \left(\alpha_{1} s_{2} p_{2}+\alpha_{2} s_{2} y_{2}\right)-\alpha_{1} s_{2} p_{2} \frac{p}{p_{2}}-\frac{\alpha_{1} s_{2} p_{2}}{F} \int_{0}^{h} \Theta(\tau) \frac{y(t-\tau)}{y_{2}} \frac{p_{2}}{p} \mathrm{~d} \tau+\alpha_{1} s_{2} p_{2}
\end{aligned}
$$




$$
\begin{aligned}
& +\frac{\alpha_{1} s_{2} p_{2}}{y_{2}} y-\frac{\rho}{(\hat{\delta}+\varepsilon) s_{1}}\left[\left(s-s_{2}\right)+\left(y-y_{2}\right)\right]\left(\hat{\delta}\left(s-s_{2}\right)+\varepsilon\left(y-y_{2}\right)\right) \\
& +\frac{\alpha_{1} s_{2} p_{2}}{F} \int_{0}^{h} \Theta(\tau) \ln \left(\frac{y(t-\tau)}{y}\right) \mathrm{d} \tau .
\end{aligned}
$$

We note that

$$
\rho\left(y-y_{2}\right)\left(1-\frac{s_{2}}{s}\right)=-\frac{\rho\left(y-y_{2}\right)}{s s_{2}}\left(s-s_{2}\right)^{2}+\frac{\rho\left(y-y_{2}\right)}{s_{2}}\left(s-s_{2}\right)
$$

Using equalities (21) in case $i=2$, we get

$$
\begin{aligned}
\frac{\mathrm{d} L_{2}}{\mathrm{~d} t}= & -\left(\hat{\delta}_{s_{2}}+\rho\left(y-y_{2}\right)+\frac{\rho \hat{\delta} s}{\hat{\delta}+\varepsilon}\right) \frac{\left(s-s_{2}\right)^{2}}{s s_{2}}-\frac{\rho \varepsilon\left(y-y_{2}\right)^{2}}{(\hat{\delta}+\varepsilon) s_{2}} \\
& -\alpha_{1} s_{2} p_{2}\left(\frac{s_{2}}{s}-1-\ln \left(\frac{s_{2}}{s}\right)\right)+\alpha_{2} s_{2} y_{2}\left(2-\frac{s_{2}}{s}-\frac{s}{s_{2}}\right) \\
& -\alpha_{1} s_{2} p_{2}\left[\frac{s p y_{2}}{s_{2} p_{2} y}-1-\ln \left(\frac{s p y_{2}}{s_{2} p_{2} y}\right)\right] \\
& -\frac{\alpha_{1} s_{2} p_{2}}{F} \int_{0}^{h} \Theta(\tau)\left[\frac{p_{2} y(t-\tau)}{p y_{2}}-1-\ln \left(\frac{p_{2} y(t-\tau)}{p y_{2}}\right)\right] \mathrm{d} \tau .
\end{aligned}
$$

Equation (25) can be simplified as:

$$
\begin{aligned}
\frac{\mathrm{d} L_{2}}{\mathrm{~d} t}= & -\left(\hat{\delta}_{s_{2}}+\rho\left(y-y_{2}\right)+\frac{\rho \hat{\delta} s}{\hat{\delta}+\varepsilon}\right) \frac{\left(s-s_{2}\right)^{2}}{s s_{2}}-\frac{\rho \varepsilon\left(y-y_{2}\right)^{2}}{(\hat{\delta}+\varepsilon) s_{2}} \\
& -\alpha_{1} s_{2} p_{2} H\left(\frac{s}{s_{2}}\right)+\alpha_{2} s_{2} y_{2}\left(2-\frac{s_{2}}{s}-\frac{s}{s_{2}}\right) \\
& -\frac{\alpha_{1} s_{2} p_{2}}{F} H\left(\frac{s p y_{2}}{s_{2} p_{2} y}\right)-\frac{\alpha_{1} s_{2} p_{2}}{F} \int_{0}^{h} \Theta(\tau) H\left(\frac{p_{2} y(t-\tau)}{p y_{2}}\right) \mathrm{d} \tau .
\end{aligned}
$$

We note that, $\frac{\mathrm{d} L_{2}}{\mathrm{~d} t} \leq 0$ when $\hat{\delta} s_{2}-\rho y_{2} \geq 0$, where $\frac{\mathrm{d} L_{2}}{\mathrm{~d} t}=0$ occurs at $\Pi_{2}$. The global asymptotic stability of $\Pi_{2}$ follows from LIP.

\section{Numerical Simulations}

This section is devoted to performing some numerical simulations for model (5)-(8). Let us choose

$$
\rho(\tau)=\tilde{\delta}\left(\tau-\tau_{1}\right)
$$

where $\tilde{\delta}($.$) is the Dirac delta function and \tau_{1} \in[0, h]$ is constant. Let $h \rightarrow \infty$, then we obtain

$$
\int_{0}^{\infty} \rho_{1}(\tau) \mathrm{d} \tau=1, \quad F=\int_{0}^{\infty} \tilde{\delta}\left(\tau-\tau_{1}\right) \mathrm{e}^{-\mu \tau} \mathrm{d} \tau=\mathrm{e}^{-\mu_{1} \tau_{1}}
$$

Moreover,

$$
\int_{0}^{\infty} \tilde{\delta}\left(\tau-\tau_{1}\right) \mathrm{e}^{-\mu_{1} \tau} y(t-\tau) \mathrm{d} \tau=\mathrm{e}^{-\mu_{1} \tau_{1}} y\left(t-\tau_{1}\right)
$$


Hence, model (5)-(8), becomes

$$
\begin{gathered}
\dot{s}(t)=\beta-\hat{\delta} s(t)-\alpha_{1} s(t) p(t)-\alpha_{2} s(t) y(t)+\rho y(t), \\
\dot{y}(t)=\alpha_{1} s(t) p(t)+\alpha_{2} s(t) y(t)-\varepsilon y(t)-\rho y(t), \\
\dot{p}(t)=m \mathrm{e}^{-\mu_{1} \tau_{1}} y\left(t-\tau_{1}\right)-\gamma p(t)-q z(t) p(t), \\
\dot{z}(t)=r z(t) p(t)-\mu z(t) .
\end{gathered}
$$

For model (26)-(29), the threshold parameters are given by:

$$
\begin{aligned}
& R_{0}=\frac{s_{0}\left(\mathrm{e}^{-\mu_{1} \tau_{1}} m \alpha_{1}+\gamma \alpha_{2}\right)}{(\varepsilon+\rho) \gamma}, \\
& R_{1}^{z}=\frac{r \mathrm{e}^{-\mu_{1} \tau_{1}} m y_{2}}{\mu \gamma}, \\
& R_{\text {Hum }}^{z}=\frac{r \mathrm{e}^{-\mu_{1} \tau_{1}} m s_{0} \delta}{\mu \varepsilon \gamma R_{0}}\left(R_{0}-1\right),
\end{aligned}
$$

where $y_{2}$ is given by Equation (14). Model (26)-(29) will be solved using the values of the parameters listed in Table 1.

Now we investigate our theoretical results given in Theorem 1-3. We consider the following two cases:

Case I: Effect of $\alpha, \mu$ and $h$ on the asymptotic behaviors of steady states:

In this case, we have chosen three different initial conditions for model (26)-(29) as follows:

Initial-1: $\left(\zeta_{1}(\eta), \zeta_{2}(\eta), \zeta_{3}(\eta), \zeta_{4}(\eta)\right)=(600,1,1,10)$, (Solid lines in the figures)

Initial-2: $\left(\zeta_{1}(\eta), \zeta_{2}(\eta), \zeta_{3}(\eta), \zeta_{4}(\eta)\right)=(200,1.5,3,5)$, (Dashed lines in the figures)

Initial-3: $\left(\zeta_{1}(\eta), \zeta_{2}(\eta), \zeta_{3}(\eta), \zeta_{4}(\eta)\right)=(90,4,9,12), \quad \eta \in(-\infty, 0]$. (Dotted lines in the figures)

Further, we fix the value of $\tau_{1}=0.2$ and we use three sets of parameters $\alpha_{1}$ and $r$ to investigate the following five scenarios.

Scenario 1: $\alpha_{1}=0.0001$ and $r=0.008$. For this set of parameters, we have $R_{0}=0.7934<1$ and $R_{1}^{z}=0.6276<1$. From Figure 1 it can be seen that the solutions with all initial conditions converge to $\Pi_{0}=(1000,0,0,0)$. This means that according to Theorem $1 \Pi_{0}$ is GAS. In this case the healthy state will be reached and the HCV particles will be removed.

Table 1. Some parameters and their values of model (26)-(29).

\begin{tabular}{cccccccc}
\hline Notation & Value & Notation & Value & Notation & Value & Notation & Value \\
\hline$\beta$ & 10 & $\rho$ & 0.01 & $q$ & 0.1 & $\mu_{1}$ & 0.1 \\
$\hat{\delta}$ & 0.01 & $\varepsilon$ & 0.5 & $r$ & Varied & & \\
$\alpha_{1}$ & Varied & $m$ & 10 & $\mu$ & 0.1 & & \\
$\alpha_{2}$ & 0.0001 & $\gamma$ & 3 & $\tau_{1}$ & Varied & & \\
\hline
\end{tabular}


Scenario 2: $\alpha_{1}=0.001$ and $r=0.001$. With such choice we get, $R_{1}^{z}=0.5538<1<R_{0}=6.1695<1+\frac{\varepsilon}{\rho}=51$ and $\Pi_{1}$ exists with $\Pi_{1}=(162.09,16.76,51.03,0)$. This result confirms Lemma 1. Theorem 2 states that, $\Pi_{1}$ is GAS and this is shown in Figure 2. This case represents the

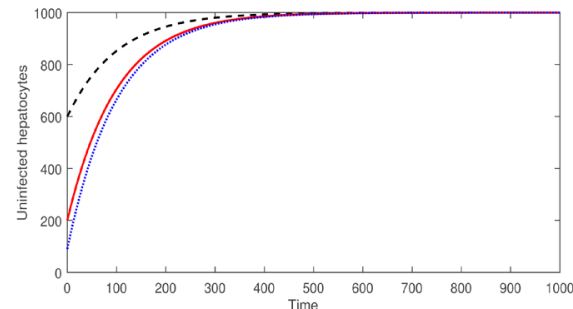

(a)

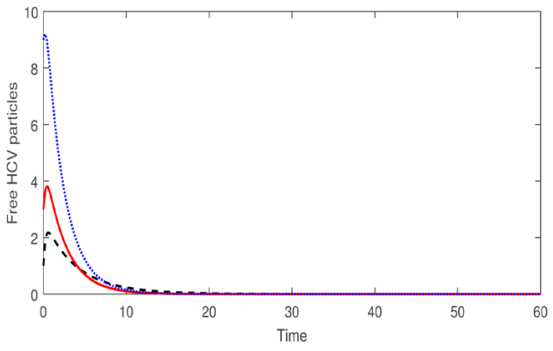

(c)

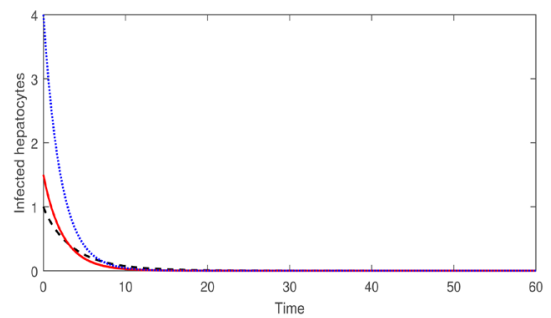

(b)

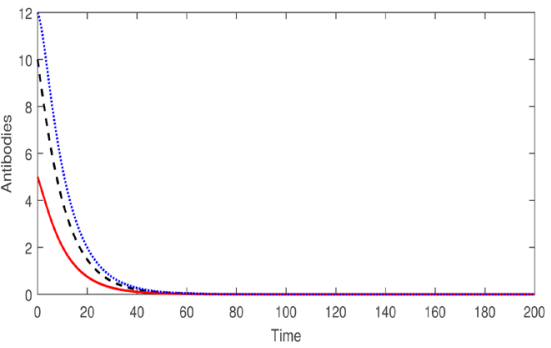

(d)

Figure 1. The simulation of trajectories of system (26)-(29) in case of $R_{0} \leq 1$. (a) The concentration of uninfected hepatocytes; (b) The concentration of infected hepatocytes; (c) The concentration of free HCV particles; (d) The concentration of antibodies.

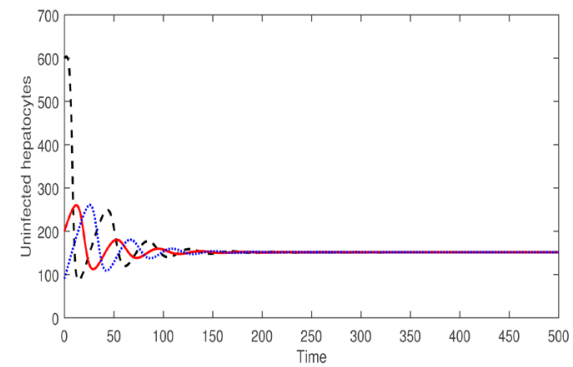

(a)

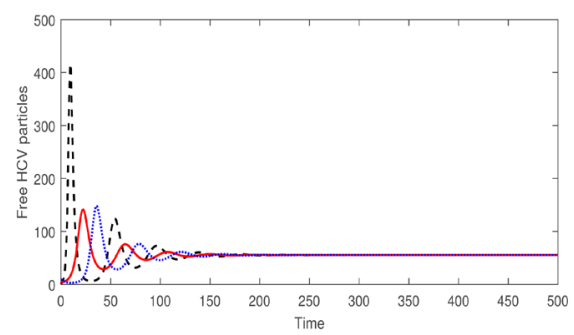

(c)

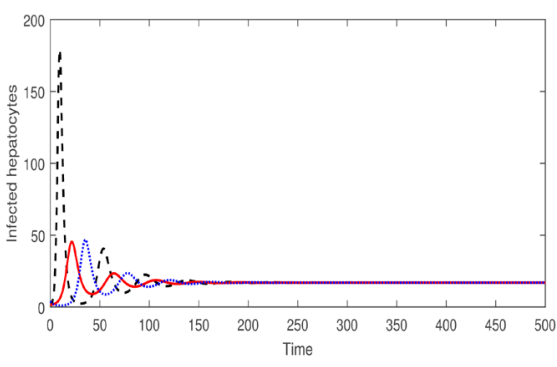

(b)

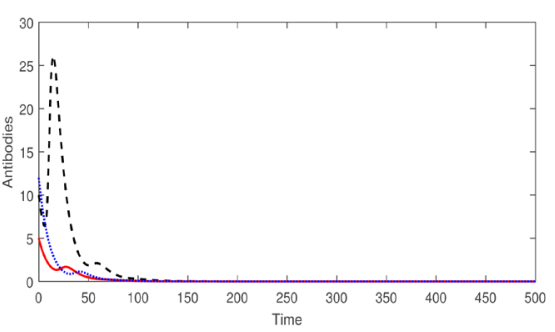

(d)

Figure 2. The simulation of trajectories of system (26)-(29) in case of $R_{1}^{z}=0.5538<1<R_{0}$. (a) The concentration of uninfected hepatocytes; (b) The concentration of infected hepatocytes; (c) The concentration of free HCV particles; (d) The concentration of antibodies. 
persistence of the HCV particles but with inactive antibody immune response.

Scenario 3: $\alpha_{1}=0.001$ and $r=0.01$. Then, we calculate $R_{0}=6.1695>1$, $R_{1}^{z}=3.1669>1$ and $\hat{\delta} s_{2}-\rho y_{2}=4.6948>0$. Lemma 1 and Theorem 3 establish that, $\Pi_{2}$ exists and it is globally asymptotically stable. From Figure 3, we find that the numerical results agree with the theoretical one presented in Theorem 3. For all initial conditions the states reach the steady state $\Pi_{2}=(480.24,10.4,10,83.74)$. This case corresponds to a chronic HCV infection with active antibody immune response.

Case II: Effect of the time delays on the free HCV particles dynamics:

Let us take the initial conditions (Initial-2). We choose the values $\alpha_{1}=0.001$ and $r=0.01$. we assume that $\tau^{*}=\tau_{1}$. Table 2 contains the values of all threshold parameters and equilibria of system (26)-(29) with different values of $\tau^{*}$.

From Table 2 we can see that, the values of $R_{0}$, and $R_{1}^{z}$ are decreased as $\tau^{*}$ is increased. Moreover, $\tau^{*}$ has a significant effect on the stability of steady states of the system. Table 2 and Figure 4 show that a high value of $\tau^{*}$

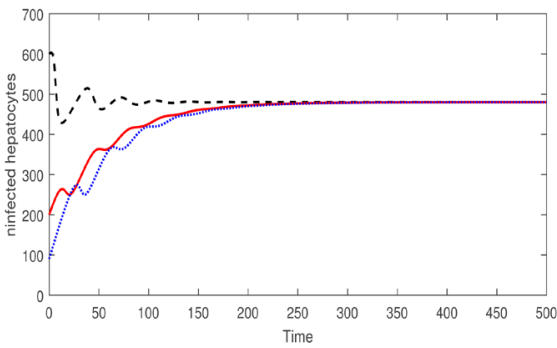

(a)

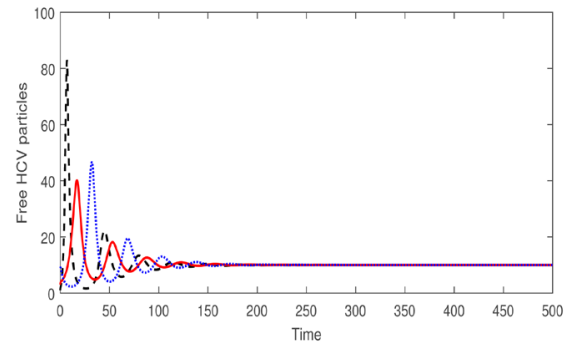

(c)

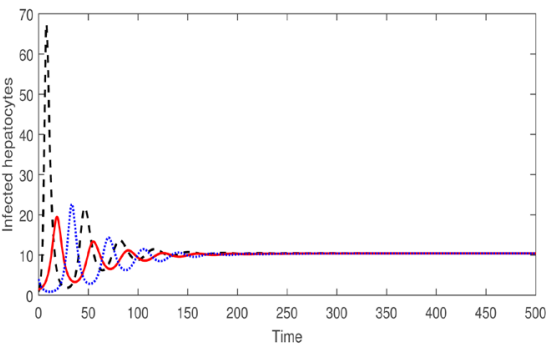

(b)

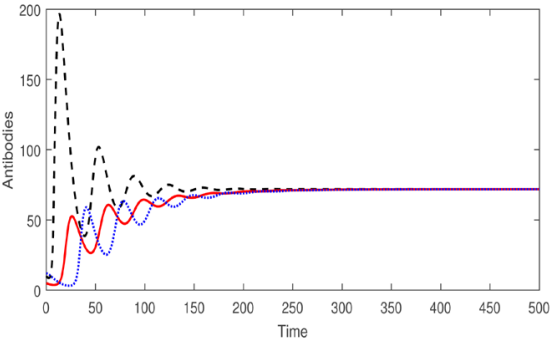

(d)

Figure 3. The simulation of trajectories of system (26)-(29) in case of $R_{1}^{z}>1$. (a) The concentration of uninfected hepatocytes; (b) The concentration of infected hepatocytes; (c) The concentration of free HCV particles; (d) The concentration of antibodies.

Table 2. The values of the threshold parameters and the equilibria of system (26)-(29) with different values of $\tau^{*}$.

\begin{tabular}{cccc}
\hline$\tau^{*}$ & $R_{0}$ & $R_{1}^{z}$ & The steady states \\
\hline 0.0 & 6.73 & 3.47 & $E_{2}=(480.24,10.4,10,74)$ \\
8 & 3.13 & 1.57 & $E_{2}=(479.88,10.42,10.01,16.8)$ \\
15 & 1.65 & 0.77 & $E_{1}=(603.61,7.73,5.76,0)$ \\
23 & 0.85 & 0.35 & $E_{0}=(1000,0,0,0,0,0)$ \\
\hline
\end{tabular}




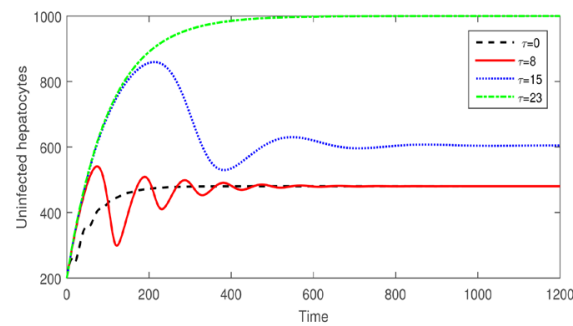

(a)

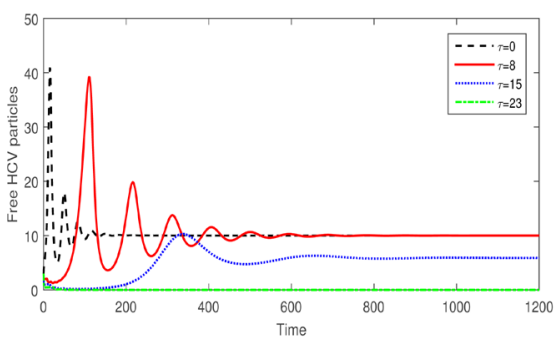

(c)

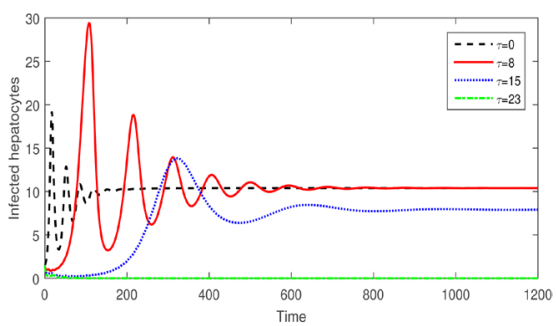

(b)

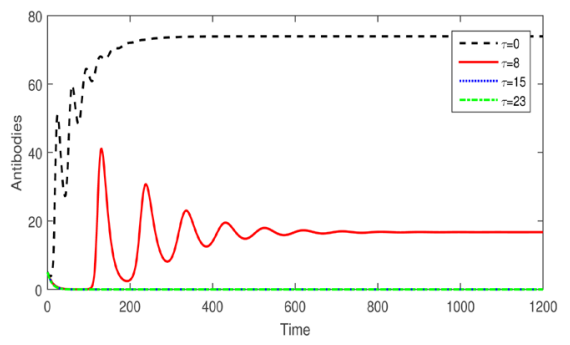

(d)

Figure 4. The effect of delays on the behaviour of all trajectories of system (26)-(29). (a) The concentration of uninfected hepatocytes; (b) The concentration of infected hepatocytes; (c) The concentration of free HCV particles; (d) The concentration of antibodies.

decreases the concentration of infected hepatocytes, free HCV particles, antibodies, and increases the population of uninfected hepatocytes. Therefore, the steady states of the system will eventually stabilized around the healthy state $\Pi_{0}$.

\section{References}

[1] Banerjee, S., Keval, R. and Gakkhar, S. (2013) Modeling the Dynamics of Hepatitisc Virus with Combined Antiviral Drug Therapy: Interferonand Rivavirin. Mathematical Biosciences, 245, 235-248. https://doi.org/10.1016/j.mbs.2013.07.005

[2] Crotty, S., Cameron, C.E. and Andino, R. (2001) RNA Virus Error Catastrophe: Direct Molecular Test by Using Ribavirin. Proceedings of the National Academy of Sciences of the United States of America, 98, 6895-6900. https://doi.org/10.1073/pnas.111085598

[3] Dahari, H., Lo, A., Ribeiro, R.M. and Perelson, A.S. (2007) Modeling Hepatitis C Virus Dynamics: Liver Regeneration and Critical Drug Efficacy. Journal of Theoretical Biology, 247, 371-381. https://doi.org/10.1016/j.jtbi.2007.03.006

[4] Dahari, H., Ribeiro, R.M. and Perelson, A.S. (2007) Triphasic Decline of Hepatitis C Virus RNA during Antiviral Therapy. Hepatology, 46, 16-21. https://doi.org/10.1002/hep.21657

[5] Dixit, N.M., Layden-Almer, J.E., Layden, T.J. and Perelson, A.S. (2004) Modelling How Ribavirin Improves Interferon Response Rates in Hepatitis C Virus Infection. Nature, 432, 922-924. https://doi.org/10.1038/nature03153

[6] Keval, R., Banerjee, S. and Gakkhar, S. (2012) Dynamics of Hepatitis C Virus (HCV) Infection with Gompertzian Proliferation. Procedia Engineering, 38, 2453-2462. https://doi.org/10.1016/j.proeng.2012.06.290

[7] Li, J., Men, K., Yang, Y. and Li, D. (2015) Dynamical Analysis on a Chronic Hepatitis C Virus Infection Model with Immune Response. Journal of Theoretical Biology, 
365, 337-346. https://doi.org/10.1016/j.jtbi.2014.10.039

[8] Reluga, T.C., Dahari, H. and Perelson, A.S. (2009) Analysis of Hepatitis C Virus Infection Models with Hepatocyte Homeostasis. SIAM Journal of Applied Mathematics, 69, 999-1023. https://doi.org/10.1137/080714579

[9] Wang, Y., Zhou, Y., Brauer, F. and Heffernan, J.M. (2013) Viral Dynamics Model with CTL Immune Response Incorporating Antiretroviral Therapy. Journal of Mathematical Biology, 67, 901-934. https://doi.org/10.1007/s00285-012-0580-3

[10] Zhang, F., Li, J., Zheng, C.W. and Wang, L. (2017) Dynamics of an HBV/HCV Infection Model with Intracellular Delay and Cell Proliferation. Communications in Nonlinear Science and Numerical Simulation, 42, 464-476. https://doi.org/10.1016/j.cnsns.2016.06.009

[11] Zhao, Y. and Xu, Z. (2014) Global Dynamics for a Delayed Hepatitis C Virus Infection Model. Electronic Journal of Differential Equations, 132, 1-18.

[12] Neumann, A.U., Lam, N.P., Dahari, H., Gretch, D.R., Wiley, T.E., Layden, T.J. and Perelson, A.S. (1998) Hepatitis C viral Dynamics In Vivo and the Antiviral Efficacy of Interferon-Alpha Therapy. Science, 282, 103-107. https://doi.org/10.1126/science.282.5386.103

[13] Elaiw, A.M., AlShamrani, N.H. and Hattaf, K. (2017) Dynamical Behaviors of a General Humoral Immunity Viral Infection Model with Distributed Invasion and Production. International Journal of Biomathematics, 10, Article ID: 1750035. https://doi.org/10.1142/S1793524517500358

[14] Elaiw, A.M., Raezah, A.A. and Hattaf, K. (2017) Stability of HIV-1 Infection with Saturated Virus-Target and Infected-Target Incidences and CTL Immune Response, International Journal of Biomathematics, 10, Article ID: 1750070. https://doi.org/10.1142/S179352451750070X

[15] Wang, X., Elaiw, A.M. and Song, X. (2012) Global Properties of a Delayed HIV Infection Model with CTL Immune Response. Applied Mathematics and Computation, 218, 9405-9414. https://doi.org/10.1016/j.amc.2012.03.024

[16] Elaiw, A.M. and Al Shamrani, N.H. (2015) Global Stability of Humoral Immunity Virus Dynamics Models with Nonlinear Infection Rate and Removal. Nonlinear Analysis. Real World Applications, 26, 161-190. https://doi.org/10.1016/j.nonrwa.2015.05.007

[17] Elaiw, A.M. and AlShamrani, N.H. (2017) Stability of a General Delay-Distributed Virus Dynamics Model with Multi-Staged Infected Progression and Immune Response. Mathematical Methods in the Applied Sciences, 40, 699-719. https://doi.org/10.1002/mma.4002

[18] Wodarz, D. (2003) Hepatitis C Virus Dynamics and Pathology: The Role of CTL and Antibody Responses. Journal of General Virology, 84, 1743-1750. https://doi.org/10.1099/vir.0.19118-0

[19] Yousfi, N., Hattaf, K. and Rachik, M. (2009) Analysis of a HCV Model with CTL and Antibody Responses. Applied Mathematical Sciences, 3, 2835-2845.

[20] Meskaf, A., Tabit, Y. and Allali, K. (2015) Global Analysis of a HCV Model with CTL, Antibody Responses and Therapy. Applied Mathematical Sciences, 9, 3997-4008. https://doi.org/10.12988/ams.2015.54334

[21] Timpe, J.M., Stamataki, Z., Jennings, A., Hu, K., Farquhar, M.J., Harris, H.J., Schwarz, A., Desombere, I., Roels, G.L., Balfe, P. and McKeating, J.A. (2008) Hepatitis C Virus Cell-Cell Transmission in Hepatoma Cells in the Presence of Neutralizing Antibodies. Hepatology, 47, 17-24. https://doi.org/10.1002/hep.21959 
[22] Xiao, F., Fofana, I., Heydmann, L., Barth, H., Soulier, E., Habersetzer, F., Doffoel, M., Bukh, J., Patel, A.H., Zeisel, M.B. and Baumert, T.F. (2014) Hepatitis C Virus Cell-Cell Transmission and Resistance to Direct-Acting Antiviral Agents. PLOS Pathogens, 10, e1004128. https://doi.org/10.1371/journal.ppat.1004128

[23] Mojaver, A. and Kheiri, H. (2016) Dynamical Analysis of a Class of Hepatitis C Virus Infection Models with Application of Optimal Control. International Journal of Biomathematics, 9, Article ID: 1650038.

https://doi.org/10.1142/S1793524516500388

[24] Dahari, H., Major, M., Zhang, X., Mihalik, K., Rice, C.M., Perelson, A.S., Feinstone, S.M. and Neumann, A.U. (2005) Mathematical Modeling of Primary Hepatitis C Infection: Noncytolytic Clearance and Early Blockage of Virion Production. Gastroenterology, 128, 1056-1066. https://doi.org/10.1053/j.gastro.2005.01.049

[25] Dubey, B., Dubey, P. and Dubey, U.S. (2016) Modeling the Intracellular Pathogen-Immune Interaction with Cure Rate. Communications in Nonlinear Science and Numerical Simulation, 38, 72-90. https://doi.org/10.1016/j.cnsns.2016.02.007

[26] Maziane, M., El Lotfi, M., Mahrouf, M., Hattaf, K. and Yousfi, N. (2016) A Delayed HIV Infection Model with Specific Nonlinear Incidence Rate and Cure of Infected Cells in Eclipse Stage. Applied Mathematical Sciences, 10, 2121-2130. https://doi.org/10.12988/ams.2016.63128

[27] Hattaf, K. and Yousfi, N. (2014) Global Stability of a Virus Dynamics Model, with Cure Rate and Absorption. Journal of the Egyptian Mathematical Society, 22, 386-389. https://doi.org/10.1016/j.joems.2013.12.010

[28] Zhou, X.Y., Song, X.Y. and Shi, X.Y. (2008) A Differential Equation Model of HIV Infection of CD4+ T-Cells with Cure Rate. Journal of Mathematical Analysis and Applications, 342, 1342-1355. https://doi.org/10.1016/j.jmaa.2008.01.008

[29] Liu, X., Wang, H., Hu, Z. and Ma, W. (2011) Global Stability of an HIV Pathogenesis Model with Cure Rate. Nonlinear Analysis. Real World Applications, 12, 2947-2961. https://doi.org/10.1016/j.nonrwa.2011.04.016

[30] Wang, K., Fan, A. and Torres, A. (2010) Global Properties of an Improved Hepatitis B Virus Model. Nonlinear Analysis: Real World Applications, 11, 3131-3138. https://doi.org/10.1016/j.nonrwa.2009.11.008

[31] Hattaf, K. and Yousfi, N. (2011) Hepatitis B Virus Infection Model with Logistic Hepatocyte Growth and Cure Rate. Applied Mathematical Sciences, 5, 2327-2335.

[32] Hattaf, K. and Yousfi, N. (2011) Dynamics of HIV Infection Model with Therapy and Cure Rate. International Journal of Tomography and Statistics, 16, 74-80.

[33] Hattaf, K. and Yousfi, N. (2012) Two Optimal Treatments of HIV Infection Model. World Journal of Modelling and Simulation, 8, 27-35.

[34] Vargas-De-Leon, C. (2012) Analysis of a Model for the Dynamics of Hepatitis B with Noncytolytic Loss of Infected Cells. World Journal of Modelling and Simulation, 8, 243-259.

[35] Hattaf, K., Yousfi, N. and Tridane, A. (2012) Mathematical Analysis of a Virus Dynamics Model with General Incidence Rate and Cure Rate. Nonlinear Analysis. Real World Applications, 13, 1866-1872. https://doi.org/10.1016/j.nonrwa.2011.12.015

[36] Tian, Y.N. and Liu, X.N. (2014) Global Dynamics of a Virus Dynamical Model with General Incidence Rate and Cure Rate. Nonlinear Analysis: Real World Applications, 16, 17-26. https://doi.org/10.1016/j.nonrwa.2013.09.002

[37] Jia, J. and Sh, X. (2016) Analysis of a Viral Infection Model with Immune Impairment and Cure Rate. Journal of Nonlinear Sciences and Applications, 9, 3287-3298. 
https://doi.org/10.22436/jnsa.009.05.115

[38] Wang, J., Lang, J. and Liu, X. (2015) Global Dynamics for Viral Infection Model with Beddington-Deangelis Functional Response and an Eclipse Stage of Infected Cells, Discrete and Continuous. Dynamical Systems Series B, 20, 3215-3233. https://doi.org/10.3934/dcdsb.2015.20.3215

[39] Wang, Y. and Liu, X. (2015) Dynamical Behaviors of a Delayed HBV Infection Model with Logistic Hepatocyte Growth, Cure Rate and CTL Immune Response. Japan Journal of Industrial and Applied Mathematics, 32, 575-593. https://doi.org/10.1007/s13160-015-0184-6

[40] Hattaf, K. and Yousfi, N. (2016) A Generalized Virus Dynamics Model with Cell-to-Cell Transmission and Cure Rate. Advances in Difference Equations, 2016, 174. https://doi.org/10.1186/s13662-016-0906-3

[41] Pan, S. and Chakrabarty, S.P. (2018) Threshold Dynamics of HCV Model with Cell-to-Cell Transmission and a Non-Cytolytic Cure in the Presence of Humoral Immunity. Communications in Nonlinear Science and Numerical Simulation, 61, 180-197. https://doi.org/10.1016/j.cnsns.2018.02.010

[42] Hale, J.K. and Verduyn Lunel, S. (1993) Introduction to Functional Differential Equations. Springer-Verlag, New York. https://doi.org/10.1007/978-1-4612-4342-7 